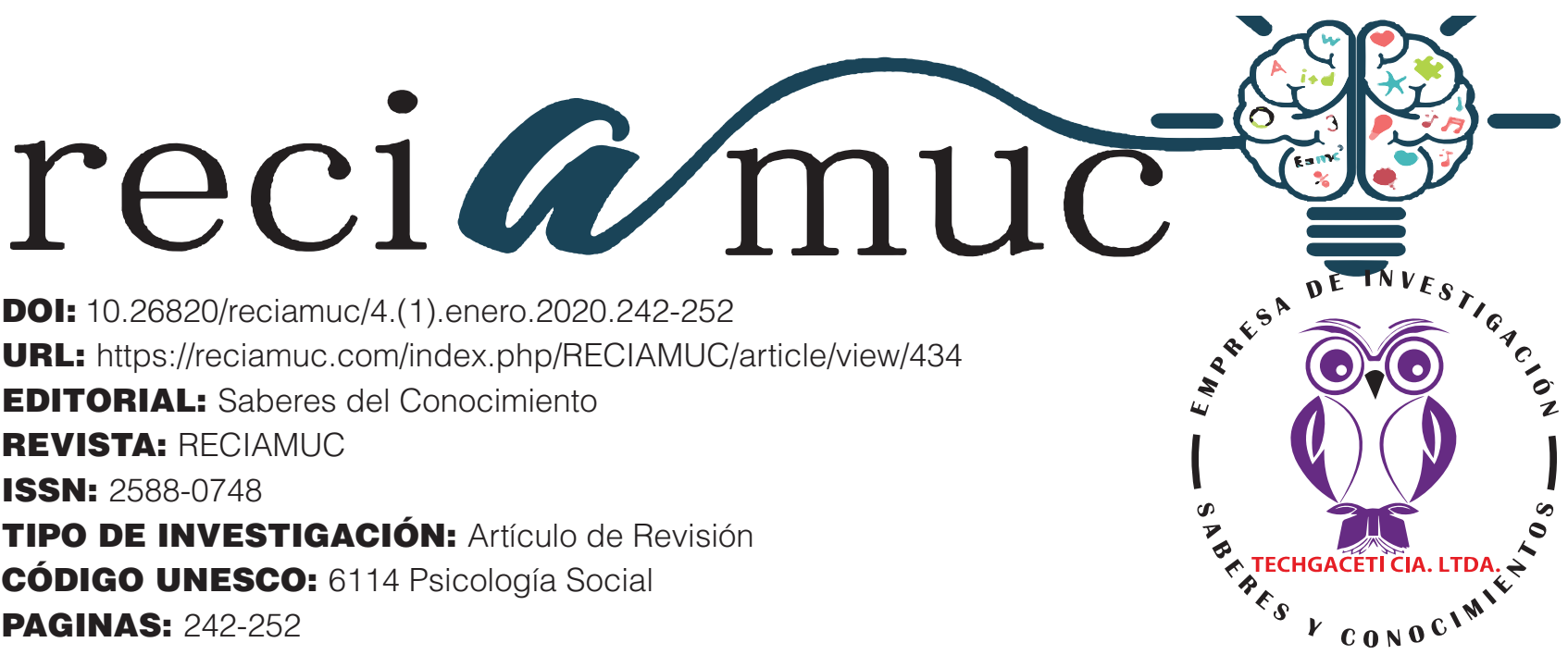

\title{
Drogadicción en la juventud estudiantil una epidemia en la actualidad
}

\section{Drug addiction in student youth an epidemic in the present}

A toxicodependência em jovens estudantes é uma epidemia no presente

\section{Martha Beatriz Baquerizo Cabrera'; Maria del Carmen Quinde2; Maria Luisa Acuña Cumba3; Ana Rosa Zambrano Bello ${ }^{4}$}

RECIBIDO: 18/11/2019 ACEPTADO: 20/12/2019 PUBLICADO: 31/01/2020

1. Especialista en Gestión de Procesos Educativos; Magister en Gerencia Educativa; Doctora en Medicina y Cirugia; Universidad de Guayaquil; Guayaquil, Ecuador; martha.baquerizoc@ug.edu.ec; (D) https://orcid.org/00000002-0852-1519

2. Magister en Psicología Clínica; Diplomado en Terapia Familiar; Magister en Terapia Familiar con Mención en Psicoterapia Sistémica; Psicóloga Clínica; Universidad de Guayaquil; Guayaquil, Ecuador; Maria.quinder@ ug.edu.ec ; (D) https://orcid.org/0000-0002-3914-826X

3. Especialista en Patología Clínica; Magister en Bioquímica Clinica; Doctora Medicina y Cirugia; Universidad de Guayaquil; Guayaquil, Ecuador; maria.acunac@ug.edu.ec; D https://orcid.org/0000-0003-1187-3109

4. Especialista en Pediatria; Magistre en Docencia Universitaria e Investigación Educativa; Doctora Medicina y Cirugia; Universidad de Guayaquil; Guayaquil, Ecuador; ana.rosaz@ug.edu.ec; D https://orcid.org/00000001-6993-482X

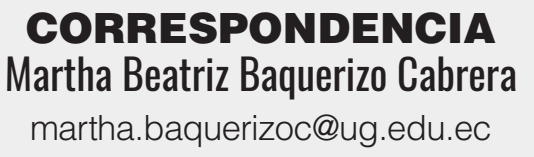

Guayaquil, Ecuador 


\title{
RESUMEN
}

La drogadicción actualmente es una problemática social a nivel mundial que produce alteraciones orgánicas en el ser humano que las ingiere, sobre todo a los más vulnerables como los adolescentes, afectando a la familia y al entorno en el que se desarrolla como el lugar donde vive o estudia y aumentando la deserción estudiantil. El objetivo de este estudio es determinar la drogadicción en la juventud estudiantil que acude al centro de investigación de la universidad de Guayaquil C.D.I.C; la metodología aplicada es de tipo observacional directa mediante entrevistas a los familiares y al joven sobre la temática. Es de corte longitudinal donde analizamos, describimos y evaluamos las variables como edad, sexo, frecuencia y tipo de consumo. Hay un predominio de la población consumidora en el sexo masculino en individuos de 14 a 17 años de edad. Además se realiza una correlación de los resultados para plantear estrategias eficaces, con la participación activa de los psicólogos y médicos en las instituciones educativas, dando prioridad orientando a los docentes, estudiantes y familiares para prevenir y disminuir esta epidemia a nivel de Ecuador.

Palabras clave: Drogadicción, Epidemia, Vulnerable, Adolescente, Universidad de Guayaquil.

\begin{abstract}
Drug addiction is currently a global social problem that produces organic alterations in the human being who ingests them, especially the most vulnerable such as adolescents, affecting the family and the environment in which it develops as the place where it lives or study and increase student desertion. The objective of this study is to determine drug addiction in the student youth that goes to the research center of the University of Guayaquil C.D.I.C; The methodology applied is of a direct observational type through interviews with family members and the young person on the subject. There is a predominance of the consuming population in the male sex in individuals 14 to 17 years. It is a longitudinal section where we analyze, describe and evaluate variables such as age, sex, frequency of consumption, type of consumption. As a conclusion of this research is to correlate the results to propose effective strategies as well as the active participation of psychologists and doctors in educational institutions giving priority to teachers, students.
\end{abstract}

Keywords: Drug addiction, Epidemic, Vulnerable, Adolescent, Guayaquil University.

\section{RESUMO}

Atualmente, a toxicodependência é um problema social global que produz alterações orgânicas no ser humano que as ingere, principalmente as mais vulneráveis, como os adolescentes, afetando a família e o ambiente em que se desenvolve como o local onde vive ou estuda e aumenta a deserção dos alunos. . O objetivo deste estudo é determinar a dependência de drogas em jovens estudantes que frequentam o centro de pesquisa da Universidade de Guayaquil C.D.I.C; A metodologia aplicada é do tipo observacional direto, por meio de entrevistas com familiares e jovens sobre o assunto. Há uma predominância da população consumidora no sexo masculino em indivíduos de 14 a 17 anos. É uma seção longitudinal onde analisamos, descrevemos e avaliamos variáveis como idade, sexo, frequência de consumo, tipo de consumo. Como conclusão desta pesquisa é correlacionar os resultados para propor estratégias efetivas, bem como a participação ativa de psicólogos e médicos em instituições de ensino, priorizando professores, alunos.

Palavras-chave: Toxicodependência, Epidemia, Vulnerável, Adolescente, Universidade de Guayaquil. 


\section{Introducción}

En la actualidad contamos con una incidencia y prevalencia que va en aumento en el Ecuador, por lo que nos enfocaremos en los pacientes en consumo de sustancias psicotrópicas quienes acuden de manera voluntaria a las instalaciones del Centro de Investigación del CDID donde se les brinda atención primaria en Salud y ayuda Psicológica, con el fin de determinar las variantes por la que los jóvenes se encuentran. Así mismo, determinaremos los tipos de drogas, sustancias contenidas entre ellas, principio activo, evaluación física, somatización del efecto por consumo de sustancias, orientación psicológica y aunada a estos elaborar planes de estrategias para disminuir el consumo de estas sustancias y en lo posible la adquisición de las mismas.

El presente estudio tiene como obetivos:

- Determinar la drogadicción en los jóvenes estudiantes que acuden al centro de investigación universitario CDIC.

- Implementar estrategias para disminuir el consumo de drogas.

- Mejorar el estado de salud y disminuir la deserción escolar.

\section{Marco teórico}

En tiempos actuales se ha observado que se está incrementando el consumo de las drogas por parte de la juventud y estudiantes universitarios, lo que es una problemática mundial, que incide en el desarrollo académico de los estudiantes disminuyendo su rendimiento, alterando su conducta, incluso pérdida de valores en la sociedad, lo que se demostrará mediante diferentes investigaciones. (Díaz-Castela, Anguiano-Garrido, \& Muela-Martínez, 2016) (Cáceres, Salazar, \& Varela, 2006)

Según el Tercer estudio epidemiológico Andino sobre el consumo de drogas en la población universitaria de Colombia 2016 manifiesta que el consumo de droga en el año mencionado es del $40 \%$, los cuales han consumido por lo menos una vez en su vida, y que la de mayor consumo es la marihuana, seguida de la LDS, que la cocaína es la droga que ocupa el tercer lugar de su consumo. (ONU, 2017) (Castaño-Castrillón, García, \& Luna, 2017)

Estudios realizados en 2014 en personas de 15 a 64 años de edad, demuestran que al menos 1 de cada 20 adultos (250 millones de personas) han consumido o consumen alguna clase de droga. Esta cifra podría equivaler a la población de Reino unido, Alemania, Francia e Italia toda unida. Se calcula que alrededor de 29 millones de personas alrededor del mundo tienen enfermedades relacionadas a este consumo. El caso más notorio lo observamos con la población consumidora mediante agujas (12 millones) del cual el 14\% está infectada con VIH. Está claro que el consumo de drogas tiene fuertes repercusiones en la salud de las personas. Para el 2014 hubo 207.400 millones de muertes por consumo de drogas es decir 43,5 muertes por cada millón de habitantes, aunque el registro sigue sin cambios notorios estos últimos años siguen siendo una cifra preocupante, la causa más común de muerte entre consumidores es la sobredosis en su mayoría relacionada con opioides. La tasa de mortalidad está estrechamente relacionada con la cantidad, uso, y tipo de droga. (ONU, 2016) (Tizoc-Marquez, Rivera-Fierro, \& Rieke-Campoy, 2017)

Encuestas realizadas en España por el plan nacional sobre drogas demuestran que el consumo de drogas ha incrementado en la actualidad comparándolo con el índice de consumo del pasado. Se presume debido a que hoy en día la droga se ha vuelto mucho más cotidiana entre los jóvenes. Según datos del estudio el $80 \%$ de los participantes admiten haber consumido alcohol durante los últimos 12 meses. En segundo y tercer lugar podemos encontrar el tabaco y la marihuana con un índice respectivamente de 20 y 30\%. En la mayoría de las encuestas se pudo definir que el patrón de consumo 
es múltiple, es decir que consumían varios tipos de drogas al mismo tiempo estas actividades se llevan comúnmente en fiestas donde es muy fácil encontrar cigarrillos, alcohol, marihuana.

El consumo de drogas puede estar ligada a múltiples factores entre los más destacables encontramos: la depresión, no tener estudios, vivir en una zona con acceso fácil a drogas, tener familiares o amigos consumidores y ser hombre. Los estudios demuestran que los hombres son mucho más propensos a caer en el mundo de las drogas, en las encuestas realizadas se detectó que el $3,57 \%$ de los varones admitieron haber probado algún tipo de droga ilegal mientras que en las mujeres el índice fue de solo $0,6 \%$. Otro dato interesante es el intervalo de tiempo en el que los encuestados admitieron haber consumido algún tipo de estupefaciente, el 2,14\% de los hombres y el $0.45 \%$ consumieron estas sustancias 2 meses previos al estudio mientas que el $1.4 \%$ de los hombres y el $0.3 \%$ consumieron estas sustancias 1 mes previo al estudio. La droga más consumida sigue siendo la marihuana con un índice de $2.4 \%$ y $0.45 \%$, luego le siguen las drogas inhalables con un $1.08 \%$ y $0.20 \%$ y en tercer lugar encontramos la cocaína con un 0.99\% y 0.22\%. (Ministerio de Sanidad, 2018)

Por otro lado, estudios realizados por el centro de monitoreo europeo para las drogas y drogadicción indican que el consumo de drogas está decayendo. Los estudios demuestran que el índice de jóvenes de 15 a 16 v consumidores de cigarrillo está en decadencia desde el 99. La cantidad de jóvenes abstemios ha incrementado en los últimos años un ejemplo de ello es Islandia con una población juvenil donde el $61 \%$ son abstemios luego sigue Suecia donde se ha incrementado de un $11 \%$ al 31\% del 2013 al 2015 incluso en países como USA el consumo de ciertas drogas como los opioides han bajado.

Las causas por las cuales las personas pueden consumir drogas son muy variadas entre ellas tenemos: el aburrimiento, amigos consumidores, por curiosidad. etc., algunas veces las drogas pueden ser parte de una cultura o de un modo de vida a este tipo de consumo se lo conoce consumo recreativo ya que forma parte de una actividad social, además muchas personas recurren a las drogas como una manera de escapar de su realidad e ignorar problemas económicos o emocionales esto puede dar pie a una adicción ya que la mayoría de las drogas son caracterizadas por ser altamente adictivas para el cuerpo humano ocasionando signos y síntomas intensos ante la abstinencia del consumo.

Además, se tiene la problemática del consumo de drogas en la niñez, la cual es una realidad cada vez más latente, estudios realizado por la administración de servicios de abuso de sustancias y salud mental muestran un índice de jóvenes de 12 a 13 años que comienzan con un consumo precoz de drogas legales como el tabaco y el alcohol, si esto persiste hasta la adolescencia el consumo se expandirá hacia otras drogas como la marihuana, los inhalantes, medicamentos de prescripción entre otros más. Para poder evitar esto es vital saber reconocer los indicios de consumo.

En la actualidad observamos un gran cambio relacionado con la edad común de los consumidores que ahora es de 23 años mientras que en los años 40 después de la guerra eran común en edades más adultas (35 a 40 años)

El Ministerio de Salud del Ecuador en el 2017, en un periodo de 5 meses atendió a 8.554 personas consumidoras. Datos de encuestas realizadas a 36.000 alumnos en centros de educación revelaron que el $12,65 \%$ de los jóvenes (de 12 a 17 años) habían consumido algún tipo de droga en el año 2015 lo que equivale a 4.554 alumnos

El problema de las drogas en los colegios de Ecuador es cada vez más preocupante según encuestas recientes el $46 \%$ de los es-

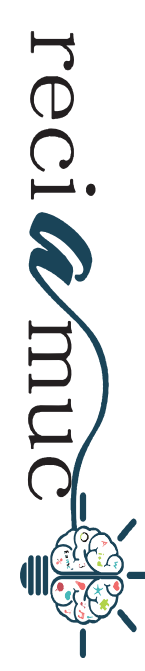


tudiantes (de 12 a 17 años) intuye que en su colegio o escuela existe el tráfico de drogas y el $34 \%$ afirma haber participado o presenciado una venta de narcóticos. Las drogas más comunes que podemos encontrar es la marihuana, el $15 \%$ de los estudiantes creen poder conseguir con facilidad esta droga, Luego le sigue la $\mathrm{H}$ con un $8 \%$, en el puesto número 3 encontramos la cocaína con una facilidad de parte de los estudiantes de un $6 \%$, éxtasis con un $4 \%$ y finalmente la pasta con un 3\% estos datos están íntimamente relacionados con el porcentaje de consumo entre los mismos estudiantes, según los datos el $29 \%$ de los estudiantes han visto a otro estudiante consumir sustancias ilegales dentro de las instalaciones del plantel. El porcentaje de consumo también depende de la región en la que viven los estudiantes ya que en zonas urbanas se encuentra más incidencia con un 34\% mientras que en el campo encontramos una incidencia de solo $18 \%$.

En cuanto a la procedencia de las drogas en Ecuador el índice de consumo de drogas licitas sigue siendo mayor al de las ilícitas hay una problemática debido a que el $60.7 \%$ de los habitantes afirman haber tenido su primer contacto en edades muy precoces (15 a 19 años). La población masculina consume más drogas licitas que las mujeres. El $63 \%$ de los hombres consumen cigarrillos mientras que solo un $29.9 \%$ de las mujeres lo hacen. El $84 \%$ de los hombres consume alcohol mientras que el $69.8 \%$ de las mujeres lo hace. El 1.7\% de los hombres consumen drogas ilícitas mientas que el 2,5\% de las mujeres lo hace. Estas cifras son preocupantemente altas y pueden ser un índice para poder desarrollar drogodependencia

Acerca del consumo por gènero, en los últimos años el consumo de drogas por sexo se ha estado equilibrando. En datos pasados se mostraba una gran brecha entre el consumo de drogas en hombres y mujeres, donde el hombre era mucho más propenso al consumo que las mujeres. Si bien actualmente los hombres siguen consumien- do más que las mujeres ya no existe una diferencia tan marcada como en el pasado debido a que las mujeres han igualado o superado el consumo de los hombres como por ejemplo en el uso de tranquilizantes donde las mujeres superan a los hombres. Este índice no solo concierne a la población en general sino también a la población estudiantil (colegiales o universitarios) donde se muestra que las mujeres tienen una incidencia muy similar a la de los hombres en el consumo de drogas licitas como el alcohol y el tabaco.

El consumo de drogas está asociado a un conjunto de efectos nefastos para la salud mental y psíquica de los jóvenes, y su bienestar a corto o largo plazo. Vinculado a un conjunto de consecuencias negativos en el ámbito educativo, tales como la falta de motivación en la escuela, el fracaso escolar o el abandono de los estudios, tiene un impacto en los esfuerzos que lleva a cabo el sector de la educación para garantizar una educación de calidad inclusiva y equitativa para todos, por ello nosotros como Facultad de Ciencias médica y representando a la salud en este proyecto, además de hacer un estudio de campo tanto cuantitativo como cualitativo hemos llegado a los jóvenes consumidores a través de campañas de prevención del consumo de las ya mencionadas sustancias psicotrópicas.

\section{Metodologia}

La metodología aplicada es de tipo observacional directa mediante entrevistas a los familiares y al joven sobre el tipo de droga, el tiempo, la frecuencia de consumo, la edad y el sexo. Esta investigación es de corte longitudinal donde analizamos, describimos y evaluamos las variables antes mencionadas.

\section{Recursos}

Se tomó en cuenta la población para la elaboración de éste estudio a los pacientes, quienes de forma voluntaria acudieron a las instalaciones del Centro de Investigación 
CDID correspondiente a la Universidad de Guayaquil.

\section{Recomendación}

- Implementar charlas informativas divididas en dos grupos tomando en cuenta a los pacientes quienes consumen y a los familiares afectados.

- Implementar estrategias dinámicas, recreativas y deportivas con el fin de mantener ocupado el tiempo de los pacientes y en lo posible la integración de los familiares.
- Determinar los factores de riesgos y posibles reincidencias en el entorno de cada paciente.

- Brindar apoyo médico continuo a través de complejos multivitamínicos con el fin de brindar las necesidades adecuadas para sobrellevar los niveles de abstinencia.

- Brindar estabilidad emocional y psicológica tanto para los pacientes afectados como para quienes conforman su entorno familiar.

- Capacitar a los pacientes y familiares y dar herramientas de manejos para sobrellevar dicha problemática.

\section{Resultados}

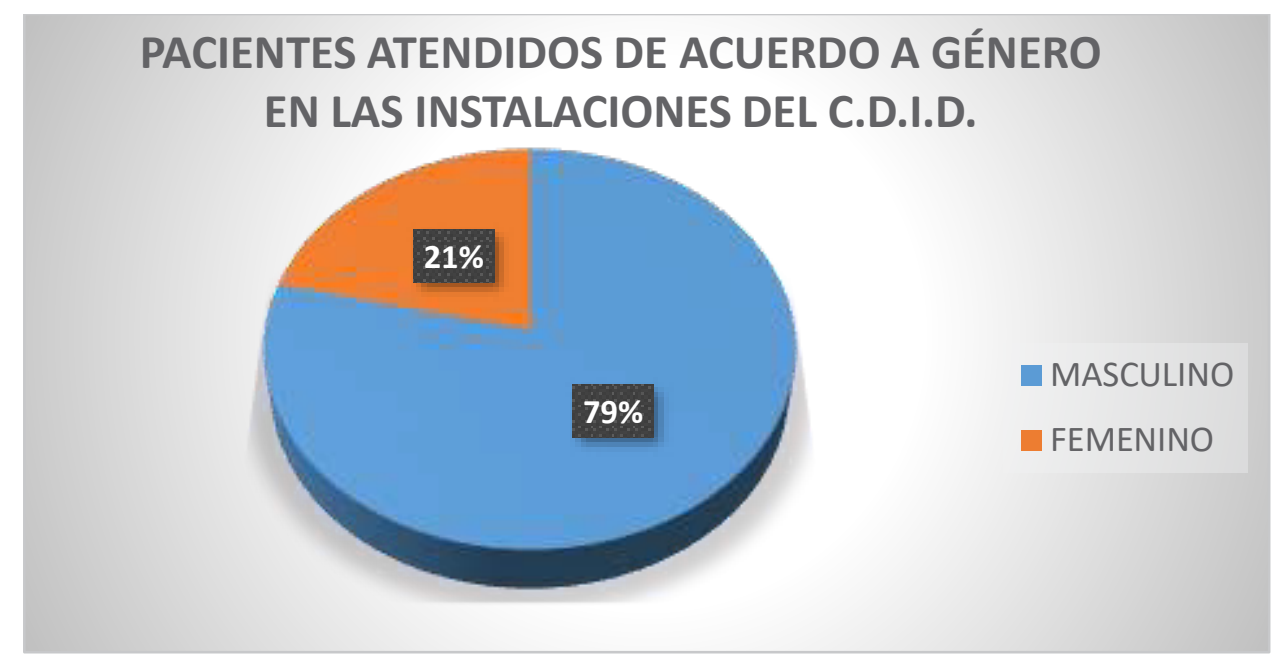

Grafico 1. Podemos observar que de la población total atendida el 79\% corresponde al género masculino, mientras que el $21 \%$ corresponde al género femenino.

Fuente: Proyecto, "Estrategias de intervención psicosocial para adolescentes vulnerables y consumidores de drogas de la zona 8" 


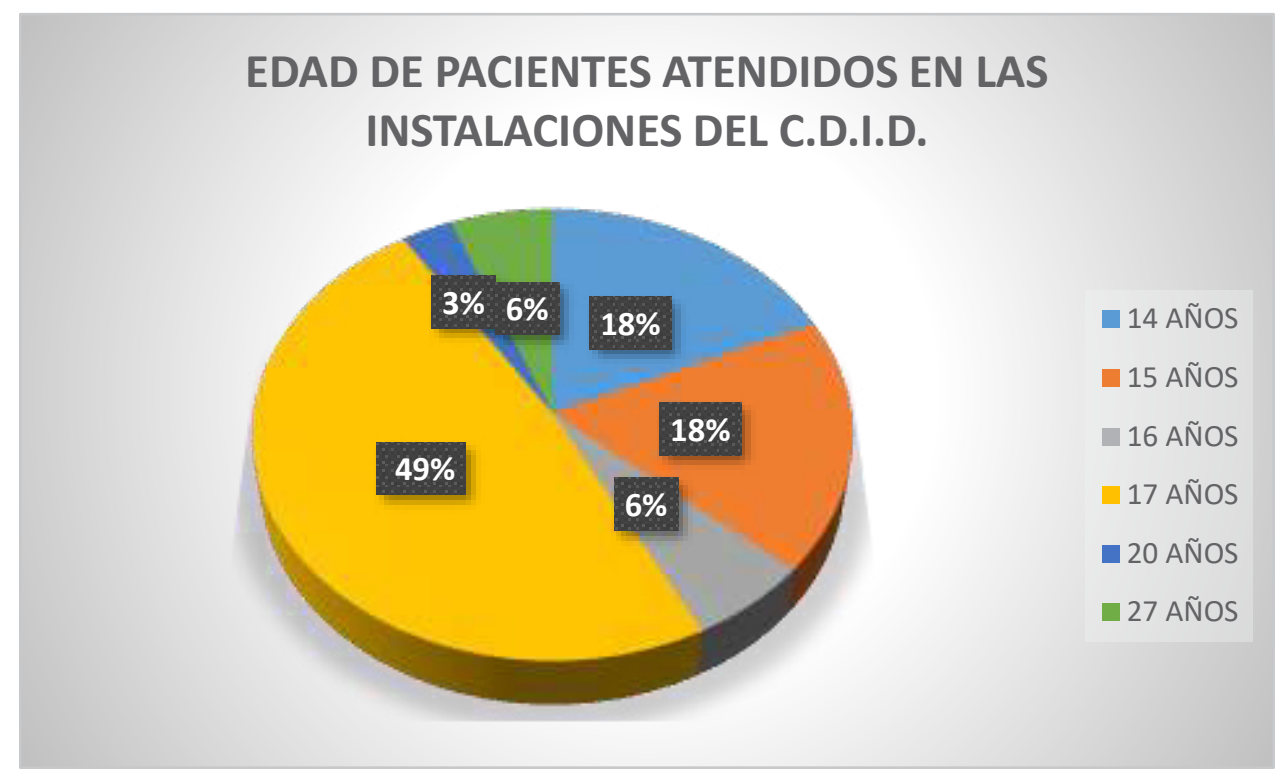

Garfico 2. Se determinó en orden cronológico de los pacientes atendidos lo siguiente: $18 \%$ en pacientes con una edad comprendida de 14 años de edad, $18 \%$ en pacientes con una edad de 15 años, $6 \%$ en pacientes con 16 años de edad, $49 \%$ en pacientes con

17 años de edad, $3 \%$ en pacientes con 20 años de edad y un 6\% en pacientes con 27 años de edad. Siendo de mayor prevalencia el consumo de drogas e pacientes con 17 años de edad con una dominancia sobre los otros resultados con un $49 \%$.

Fuente: Proyecto, "Estrategias de intervención psicosocial para adolescentes vulnerables y consumidores de drogas de la zona 8"

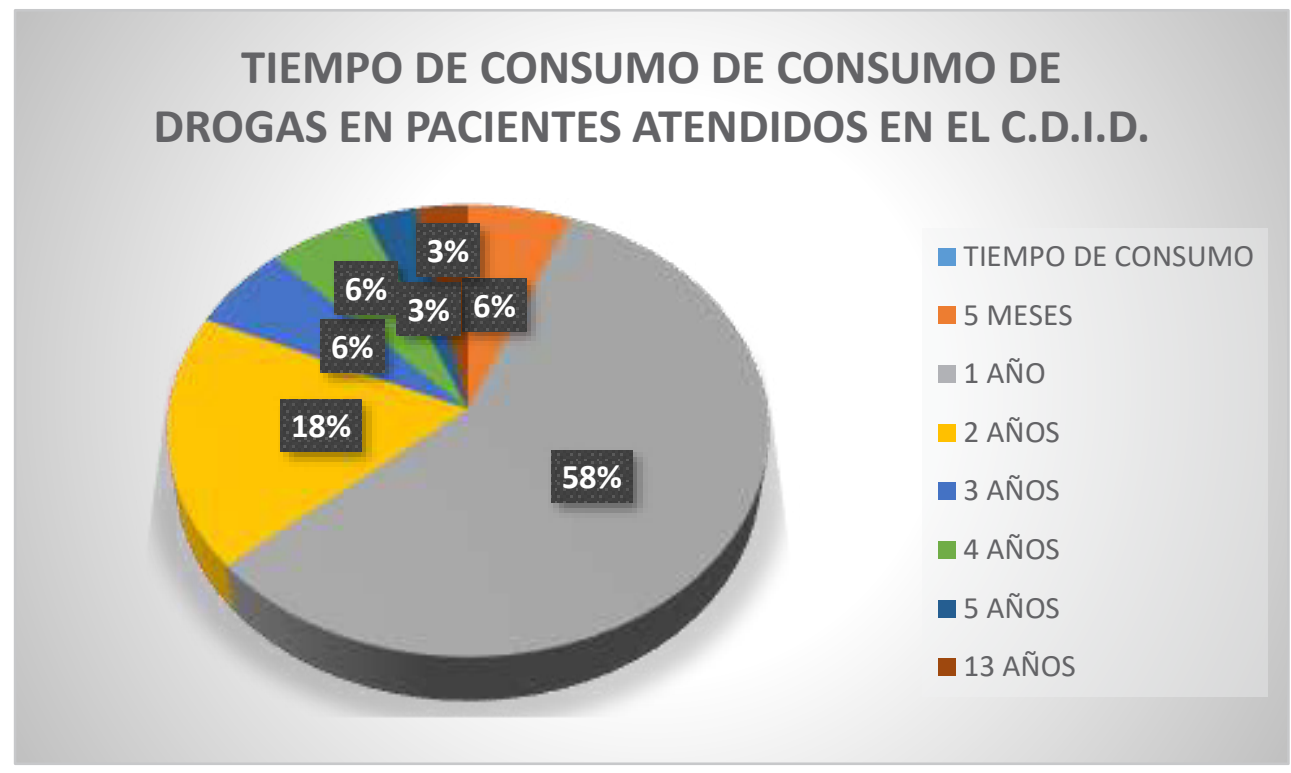

Garfico 3. De acuerdo al tiempo de consumo de drogas hasta la fecha de atención, se determinó que el $6 \%$ tiene un consumo de 5 meses, $58 \%$ en pacientes con un año de consumo, $18 \%$ en pacientes con 2 años de consumo, $6 \%$ en pacientes con 3 años de consumo, $6 \%$ en pacientes con 4 años de consumo, $3 \%$ en pacientes con 5 años de consumo y $3 \%$ en pacientes con 13 años de consumo. Se obtuvo una prevalencia del $58 \%$ de los pacientes atendidos por consumo de drogas con un periodo de 1 año.

Fuente: Proyecto, "Estrategias de intervención psicosocial para adolescentes vulnerables y consumidores de drogas de la zona 8" 


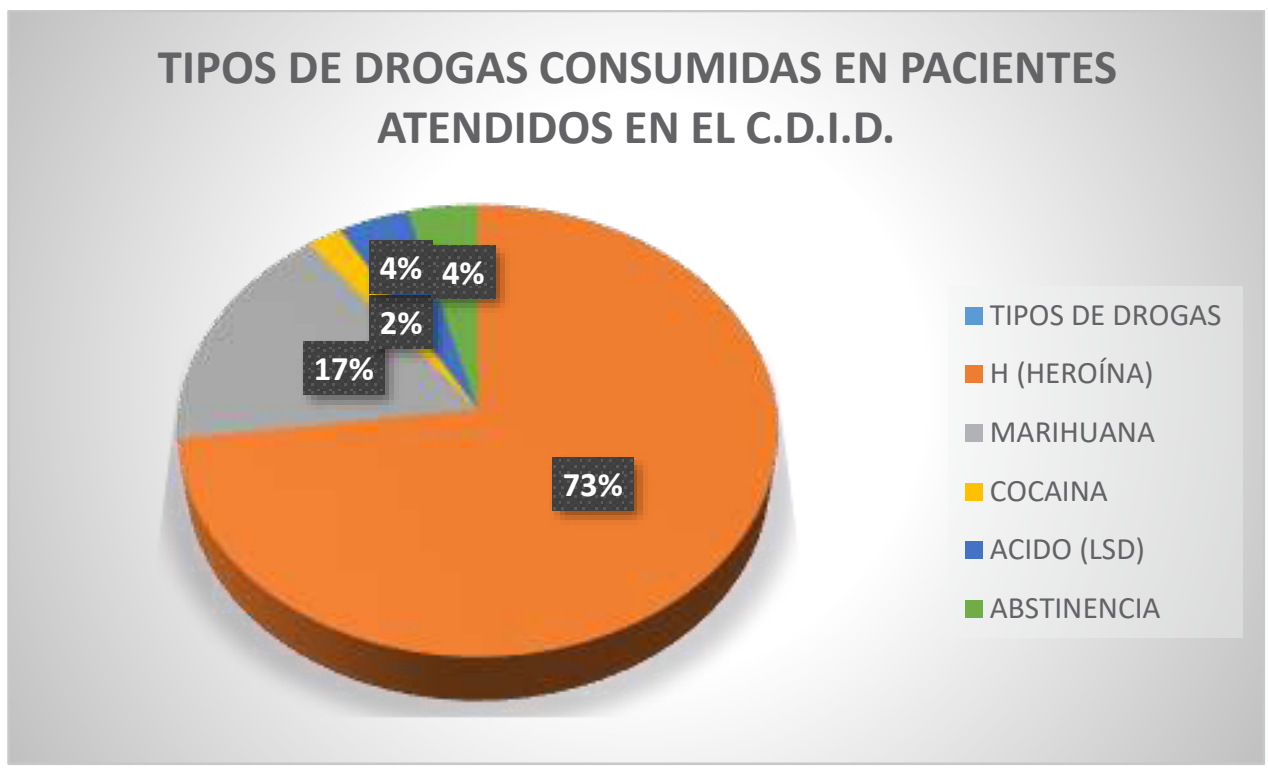

Garfico 4. Los tipos de drogas utilizados por los pacientes correspondieron al siguiente resultado: 73\% para el consumo de H (Heroína), 17\% para el consumo de Marihuana, 2\% para el consumo de Cocaína, 4\% para el consumo de Ácido (LSD), mientras que un 4\% se encuentra en un periodo de abstinencia. Se determinó que de los pacientes atendidos en las instalaciones del CDID tiene una prevalencia en el consumo de la droga $\mathrm{H}$ (Heróna).

Fuente: Proyecto, "Estrategias de intervención psicosocial para adolescentes vulnerables y consumidores de drogas de la zona 8"

\section{TIPO DE CONSUMO POR MEZCLA DE DROGAS EN PACIENTES ATENDIDOS EN EL C.D.I.D.}
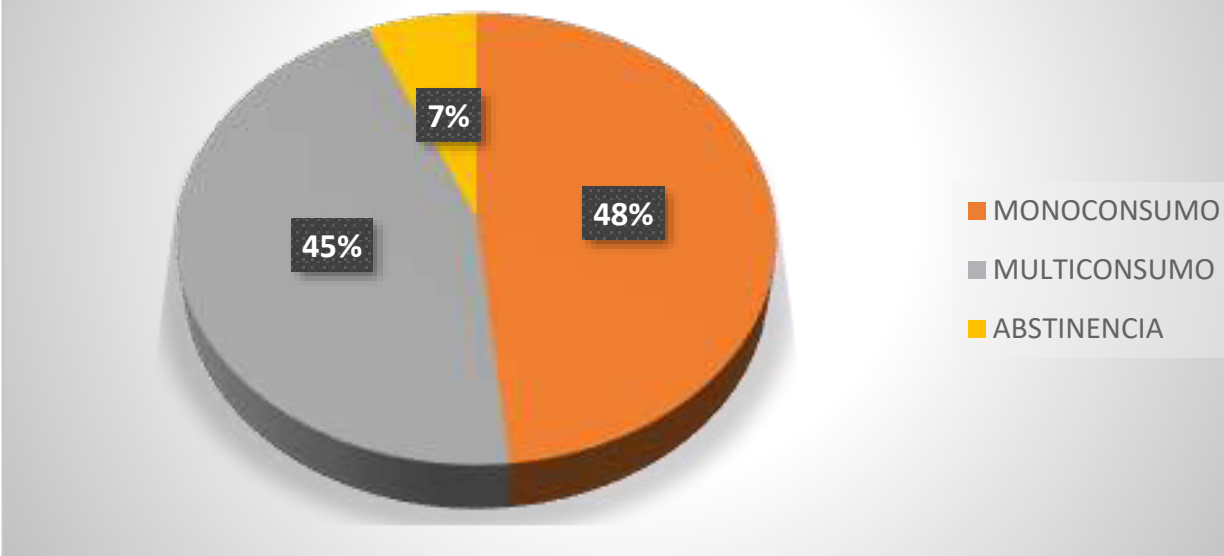

- MULTICONSUMO

ABSTINENCIA

Garfico 5. Se determinó que de los pacientes atendidos dentro de las instalaciones, para relación de sustancias es de: 48\% en el Monoconsumo de sustancias psicotrópicas, $45 \%$ en Multiconsumo y $7 \%$ en estado de abstinencia.

Fuente: Proyecto, "Estrategias de intervención psicosocial para adolescentes vulnerables y consumidores de drogas de la zona 8" 


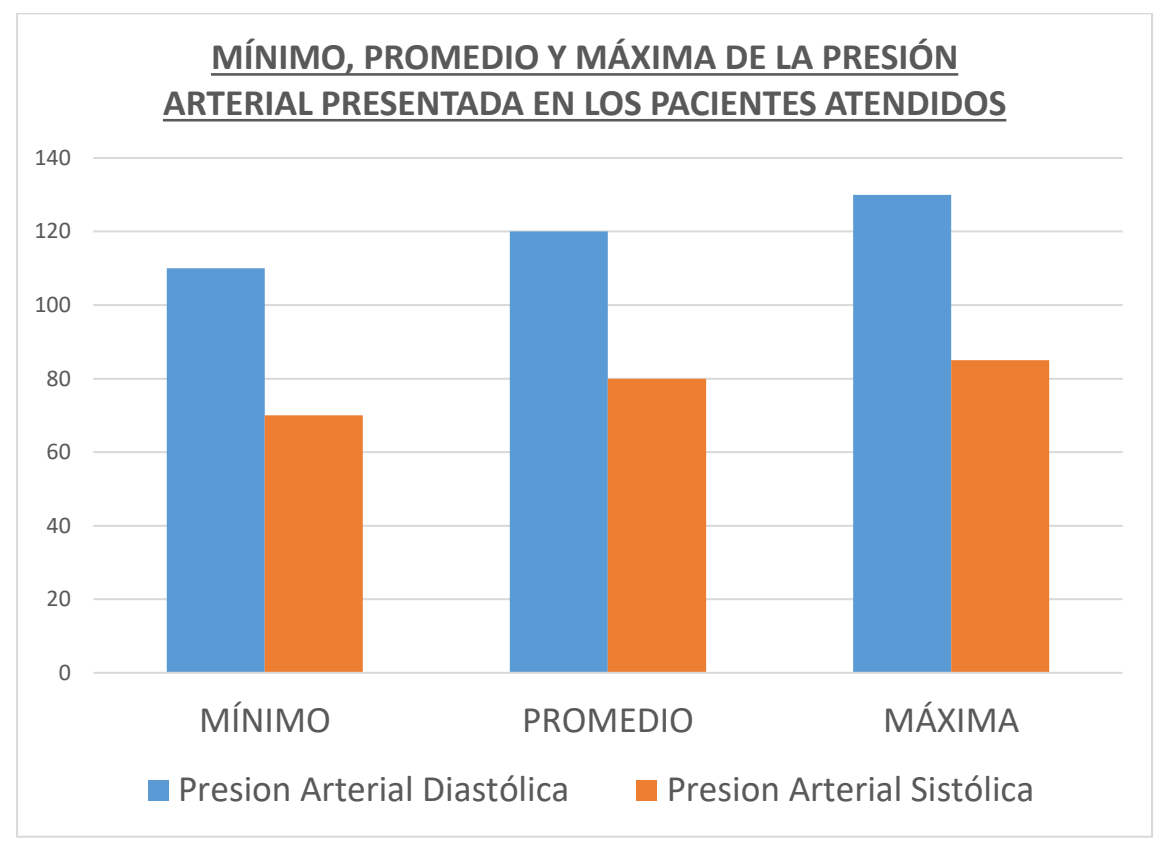

Garfico 6. De los parámetros evaluados se determinó una Mínima de Presión Arterial Diastólica 110 y Presión Arterial Sistólica de 70, un Promedio de Presión Arterias Diastólica 120 y Presión Arterial Diastólica 80 y una Máxima de Presión Arterial Diastólica de 130 y Presión Arterial Diastólica 90.

Fuente: Proyecto, "Estrategias de intervención psicosocial para adolescentes vulnerables y consumidores de drogas de la zona 8"

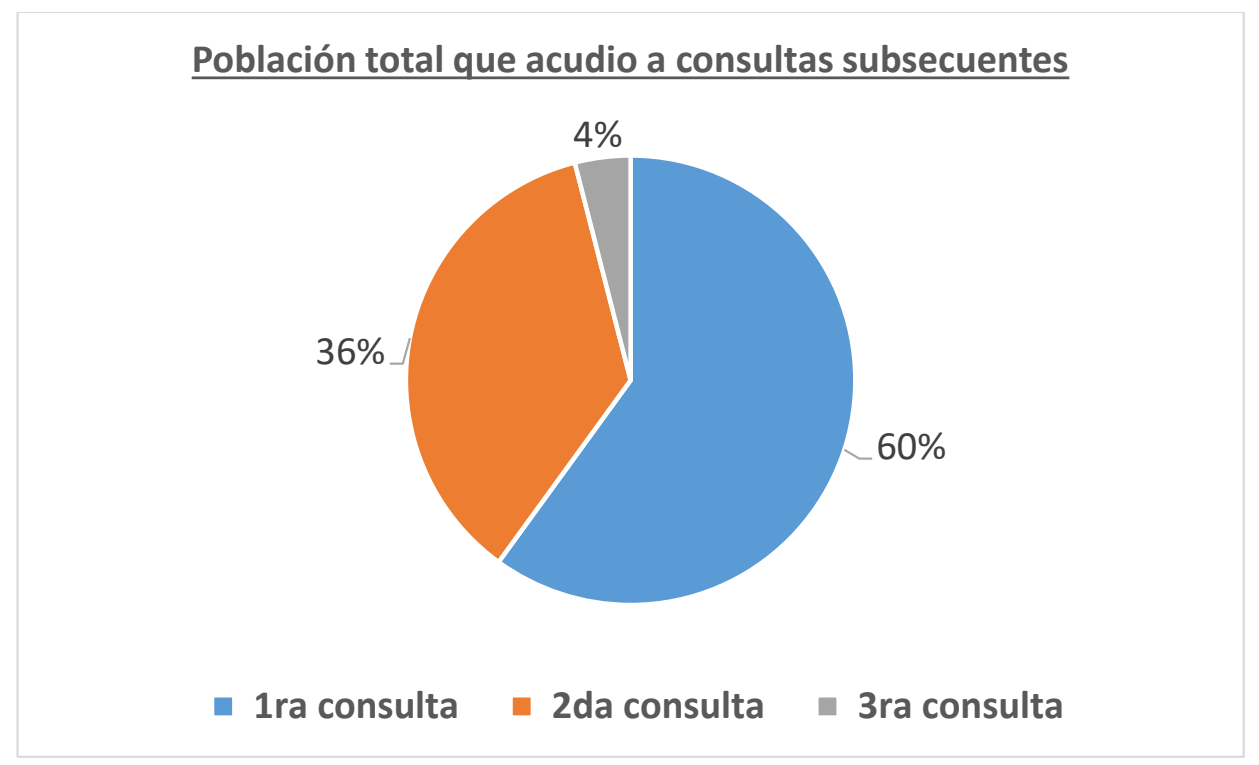

Garfico 7. Se determinó que en las consultas subsecuentes se obtuvo lo siguiente: $60 \%$ de los pacientes acudieron a 1 consulta subsecuente, el 36\% asistió a segunda consulta subsecuente y el $4 \%$ a tercera consulta subsecuente. Observamos que se da una desmotivación continua en las consultas subsecuentes por lo que se debe determinar la problemática y pronta resolución en pro a la mejora del estado de salud y control de los pacientes atendidos por problemática de consumo de sustancias psicotrópicas.

Fuente: Proyecto, "Estrategias de intervención psicosocial para adolescentes vulnerables y consumidores de drogas de la zona 8" 


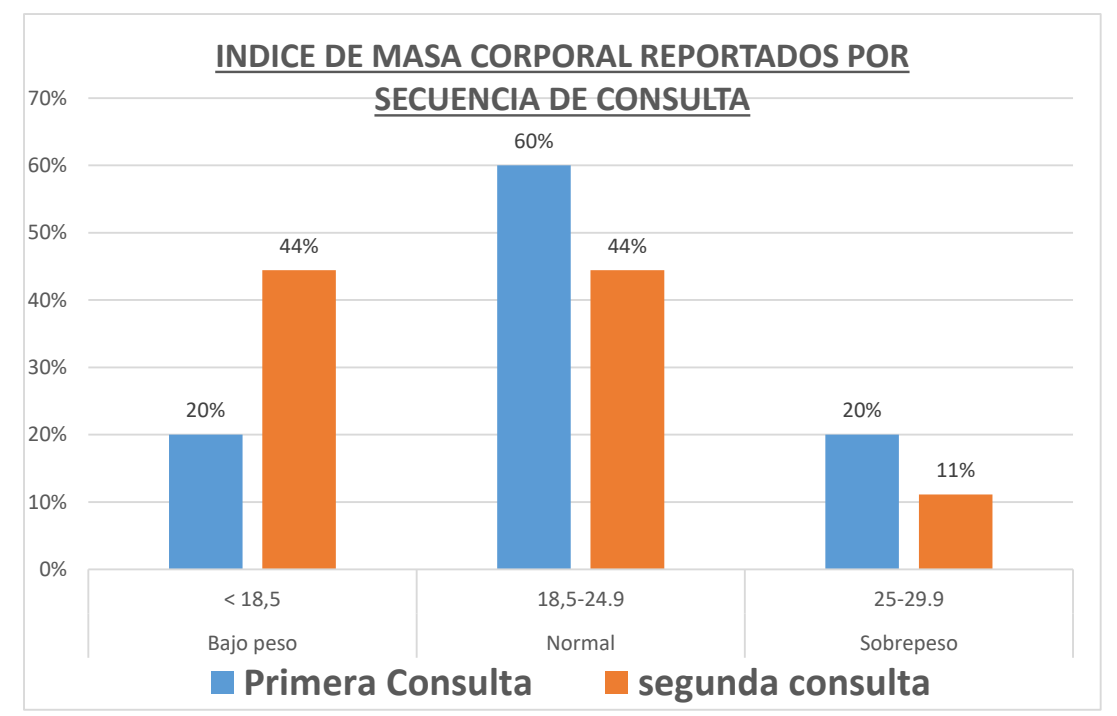

Garfico 8. De acuerdo al Índice de Masa Corporal (IMC) se pudo determinar que: en la primera consulta de la población total atendida encontramos un 20\% con un IMC bajo, un $60 \%$ con un IMC normal y $20 \%$ con IMC correspondiente a Sobrepeso. En la Segunda Consulta subsecuente se obtuvo que de los pacientes atendidos encontramos un $44 \%$ con un bajo IMC, un $44 \%$ con IMC normal y un $12 \%$ con soberpeso de acuerdo al IMC.

Fuente: Proyecto, "Estrategias de intervención psicosocial para adolescentes vulnerables y consumidores de drogas de la zona 8"

\section{Conclusiones}

En conclusión antes esta problemática es importante aplicar leyes más estrictas como derogar la ley que aprueba la tabla de consumo permisible en el Ecuador, que es lo que permite el consumo libremente, hay instituciones preocupados por esta problemática como el Centro de Investigaciones de la Universidad de Guayaquil que se encuentran haciendo talleres de capacitación y orientación a los jóvenes drogadictos, con el fin de conseguir que los estudiantes retomen los estudios y puedan salir del mundo de las drogas.

Es importante recalcar que la alcaldesa de la ciudad de Guayaquil está haciendo promociones de prevención y tratamiento actualizados a los jóvenes que se encuentran en esta problemática.

\section{Bibliografía}

Cáceres, D., Salazar, I., \& Varela, M. (2006). Consumo de drogas en jóvenes universitarios y su relación de riesgo y protección con los factores Psico- sociales. Univ. Psychol. Bogotá (Colombia), 5(3), 521-534. Obtenido de https://revistas.javeriana. edu.co/index.php/revPsycho/article/view/458

Castaño-Castrillón, J., García, S., \& Luna, J. (2017). Estudio de factores asociados y prevalencia de consumo de sustancias psicoactivas ilegales en estudiantes de una universidad colombiana. Revista de la Facultad de Medicina, 65(1), 23-30. doi:10.15446/revfacmed.v65n1.56471

Díaz-Castela, M., Anguiano-Garrido, B., \& Muela-Martínez, J. (2016). El consumo de las drogas por parte de la juventud y estudiantes universitarios. Acción Psicológica, 13(1), 53-66. doi:10.5944/ ap.13.1.16723

Ministerio de Sanidad. (2018). La Encuesta sobre uso de drogas en Enseñanzas Secundarias en España, ESTUDES. México: Plan Nacional sobre Drogas.

ONU. (2016). INFORME MUNDIAL SOBRE LAS DROGAS. Washington: ONODC. Obtenido de https://www.unodc.org/doc/wdr2016/WDR_2016_ ExSum_spanish.pdf

ONU. (2017). III Estudio epidemiológico andino sobre consumo de drogas en la población universitaria, Informe Regional, 2016. Lima - Perú: UNODC Perú y Ecuador. Obtenido de https://www.unodc. 
org/documents/peruandecuador//Informes/Otros/ Informe_Universitario_Regional.pdf

Tizoc-Marquez, A., Rivera-Fierro, K., \& Rieke-Campoy, U. (2017). REDUCCIÓN DE DAÑOS COMO ESTRATEGIA PARA EL USO Y ABUSO DE SUSTANCIAS EN MÉXICO: DESAFÍOS Y OPORTUNIDADES. Ra Ximhai, 13(2), 39-51. Obtenido de https://www.redalyc.org/pdf/461/46154510003.pdf

\section{CITAR ESTE ARTICULO:}

Baquerizo Cabrera, M., Quinde, M., Acuña Cumba, M., \& Zambrano Bello, A. (2020). Drogadicción en la juventud estudiantil una epidemia en la actualidad. RECIAMUC, 4(1), 242-252. doi:10.26820/reciamuc/4.(1).enero.2020.242-252

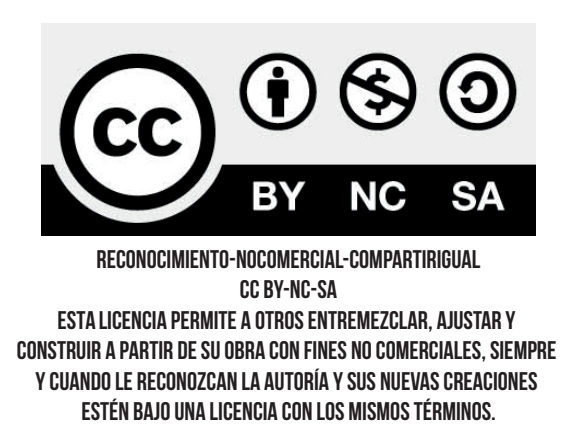

